\title{
Characterization and Localization of Power Quality disturbances Based on S-transform and Fuzzy Expert System
}

\author{
P.Kalyana Sundaram ${ }^{1}$, R.Neela ${ }^{2}$, \\ ${ }^{1}$ Assistant Professor, Electrical Engineering, Annamalai University \\ ${ }^{2}$ Professor, Electrical Engineering, Annamalai University
}

\begin{abstract}
This paper presents a new technique to characterize and localize the various types of power quality $(P Q)$ disturbances using S-Transform and Fuzzy expert system.The S-Transform method is used to extract the two input features (Standard deviation and Variances) from the distorted voltage waveforms simulated using parametric equations. The extracted features are given as input to Fuzzy expert system for the classification of various types of power quality disturbances. The results clearly indicate that the proposed method has the ability to classify the power quality disturbances with great accuracy. The performance of the proposed technique is compared with the S-Transform based neural classifier.
\end{abstract}

Keywords: Power quality, Power quality disturbances, S-transform, Fuzzy logic, Fuzzy expert system.

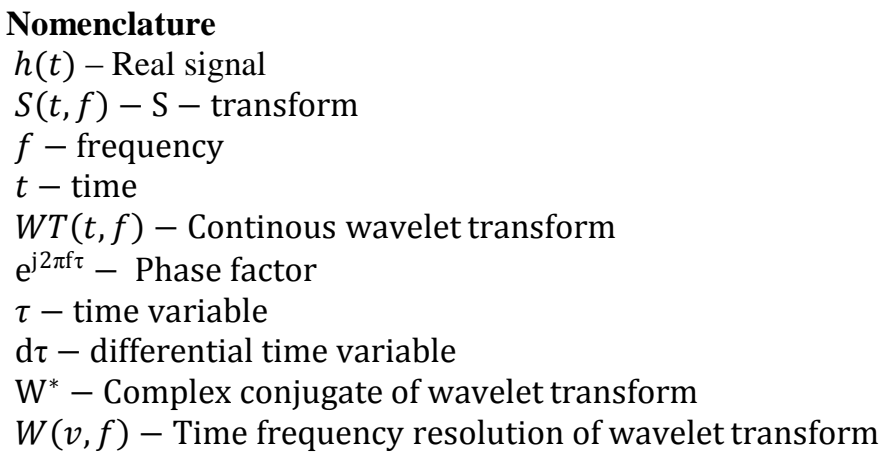

\section{Introduction}

A wide variety of power quality detection and classification tools were developed over the past few decades and are being constantly improved with a view of making them more flexible so as to analyze stationary and non stationary signals and a wide range of comparatively newer classes of disturbances such as notches and flickers, in both time and frequency domain. The various types of power quality disturbances were detected and classified using wavelet transform analysis as illustrated in [1]. The multi resolution wavelet has been presented in [2] to analyze the electromagnetic power system transients. Power quality analysis of transient signals using wavelet transform has been illustrated in [3]. Short Time Fourier Transforms (STFT) based power frequency harmonic analyzer has been discussed in [4] for the non stationary signals. The windowed FFT which is the time windowed version of Discrete Fourier transform has been applied for power quality analysis to classify a variety of disturbances in [5]. Wavelet multi resolution analysis based neural network classifier is presented in [6] for the detection and extraction of power quality disturbances. An automated online power quality disturbances classification using wavelet based pattern recognition technique has been illustrated in [7].

As wavelet transforms cannot be applied for the analysis of non stationary signals, S-transforms were implemented due to their excellent frequency resolution characteristics. Application of s-transform for power quality analysis has been discussed in [8] and a fuzzy logic based pattern recognition system along with multi resolution S-transform for power quality event classification has been discussed in [9]. A two dimensional representation of analysis of various types of power quality disturbances using DWT decomposition technique has been implemented in [10]. Wavelet based neural network classifier based on learning vector quantization network and a decision making scheme has been presented in [11]. Classification of in both single and multiple Power quality disturbance using S-transform and Pattern recognition techniques has been elaborated in [12].

An S-transform based probabilistic neural network has been presented in [13] and this combines the frequency resolution characteristics of $\mathrm{S}$ transform with the pattern recognizing ability of a neural network. Modified S-transform along with fuzzy C-means clustering technique and Particle swarm optimization has been implemented for non stationary power disturbances classification in [14]. The binary feature matrix of the system has been designed using Fourier and S-transform and a rule base has been formulated to classify the 
power quality events in [15]. The assessment of electric power quality events based on Hilbert transform along with empirical mode decomposition technique has been presented in [16].

As Hilbert transform shows greater immunity towards noise, it has been used for the detection and classification of power quality events along with ANN in [17]. A representative quality power vector has been derived for power quality analysis through an adaptive neuro fuzzy interface system in [18]. A combination of linear Kalman filter and fuzzy expert system has been used for the analysis of power quality events in [19] wherein the signal noise is estimated using a block of DWT. The windowed Hilbert Huang Transform (HHT) used for the analysis of non-stationary signal in power quality analysis has been discussed in [20]. An Stransform based MLP neural network classifier for power quality analysis has been presented in this paper.

\section{Proposed Method}

The proposed method has two stages namely

i. Feature extraction using S-transform.

ii. Classification stage using Fuzzy expert system.

S-transform is used for extracting features such as variances and standard deviation. Fuzzy expert system is used for classifying the power quality disturbances. A set of parametric equations generates the disturbance waveforms.

\subsection{Feature Extraction Stage using S-transform}

The S-transform is the generalization of the Short-time Fourier transform (STFT) and an extension of the continuous wavelet transforms (CWT). The S-transform will perform multi-resolution analysis (MRA) on a time varying power signal, as its window width varies inversely with the frequency. The main function of the $S$ transform is a Gaussian modulation cosinusoid. The output of the $\mathrm{S}$-transform is an $\mathrm{N} \times \mathrm{M}$ matrix called the $\mathrm{S}$ matrix whose rows pertain to the frequency and columns to time.

A spectrogram or sonogram, is a visual representation of the spectrum of frequencies. Spectrograms are usually created in one of the two ways. Either they are approximated as a filter bank that results from a series of band pass filters, or calculated from the time signal using the short-time Fourier transform (STFT). These two methods actually form two different time-frequency distributions, but are equivalent under some conditions. Stransform using spectrogram analysis provides better visual analysis of the signal.

The S-transform of a signal $h(t)$ is defined as

$$
\begin{aligned}
& S(t, f)=\int_{-\infty}^{\infty} h(\tau) w^{*}(\tau-t, f), e^{-j 2 \pi f \tau} d \tau \\
& \text { Where } S(t, f)=\frac{|f|}{\alpha \sqrt{2} \pi}, e^{-t f^{2} / 2 \alpha^{2}}
\end{aligned}
$$

The integration of S-transform over time results in the Fourier spectrum which is represented as

$$
H(f)=\int_{-\infty}^{\infty} S(t, f) d t
$$

For the gaussian window $\int_{-\infty}^{\infty} S(t, f) d t=1$

The original signal can be obtained from $S$-transform as

$$
h(t)=\int_{-\infty}^{\infty}\left\{\int_{-\infty}^{\infty} S(\tau, f) d \tau\right\} e^{j 2 \pi f \tau} d f
$$

The S-transform is also represented as an amplitude and phase correction of the continuous wavelet transform

$$
S(t, f)=\frac{\sqrt{f}}{2 \pi \alpha} e^{j 2 \pi f \tau}, W T(t, f)
$$

Wavelet transform is given as $W T(t, f)=\sqrt{\frac{|f|}{\alpha}}, e^{-t^{2} f^{2} / 2 \alpha^{2}}, e^{j 2 \pi f \tau}$

The $\mathrm{S}$-transform is now expressed in time frequency resolution as

$$
\begin{gathered}
S(t, f)=\int_{-\infty}^{\infty} H(v, f) w^{*}(v, f), e^{j 2 \pi v \tau} d v \\
w(v, f)=e^{-2 \pi^{2} \alpha^{2} v^{2} / t^{2}}
\end{gathered}
$$

The amplitude and the envelop mean value are given as

$$
\begin{gathered}
A(t)=\frac{\sqrt{f}}{2 \pi \alpha} \\
\tilde{A}(t)=T^{-1} \int_{0}^{T} A(t) d t
\end{gathered}
$$

The variance of the signal is the first input to fuzzy system. It is directly computed from the envelop mean value as follows

$$
V_{a r}(t)=(S(t, f)-\tilde{A}(t))^{2}
$$

The standard deviation is the second input to fuzzy system. It is obtained from the following relationship as given below

$$
S_{t d}(t)=\sqrt{V_{a r}(t)}
$$


Characterization and Localization of Power Quality disturbances Based on S-transform and Fuzzy..

\subsection{Fuzzy Expert System}

Fuzzy system provides a simple way to get definite conclusion based upon ambiguous inputs. The mamdani type of fuzzy inference system used to perform the classification of the PQ events. It has two inputs and generates one output based on 25 rules. The first input to the system is the value of standard deviation. The input is divided into five trapezoidal membership functions namely VSTD (very small standard deviation), SSTD (small standard deviation), NSTD (normal standard deviation), LSTD (large standard deviation), and VLSTD (very large standard deviation).

The second input to the system is the value of variances. It is broken into five triangular membership functions namely VV (very small variance), SV (small variance), NV (normal variance), LV (large variance), and VLV (very large variance). The fuzzy expert system is shown in figure 1

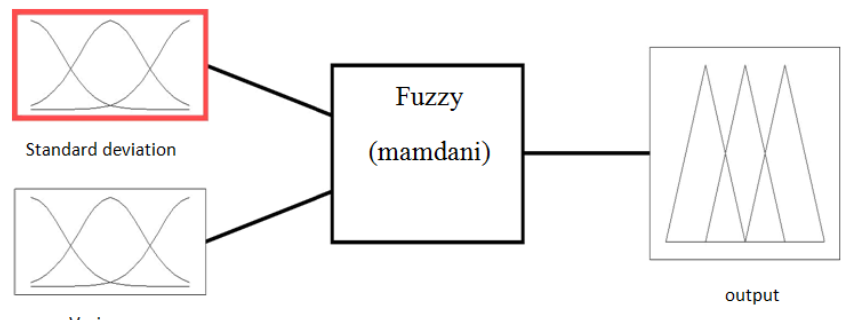

Figure 1.Fuzzy expert system

The output memberships function and rule viewers of fuzzy expert system are shown in figure 2 and figure 3 .

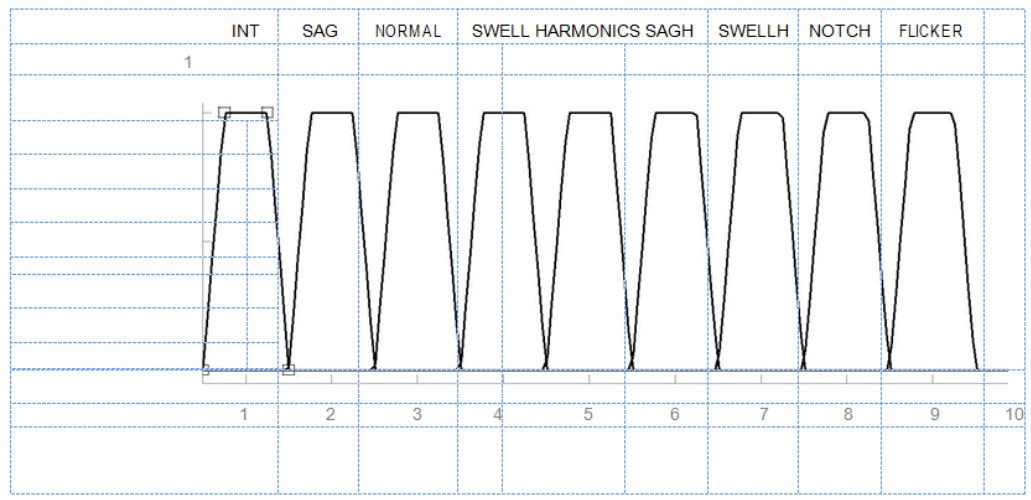

Figure 2.Output membership function

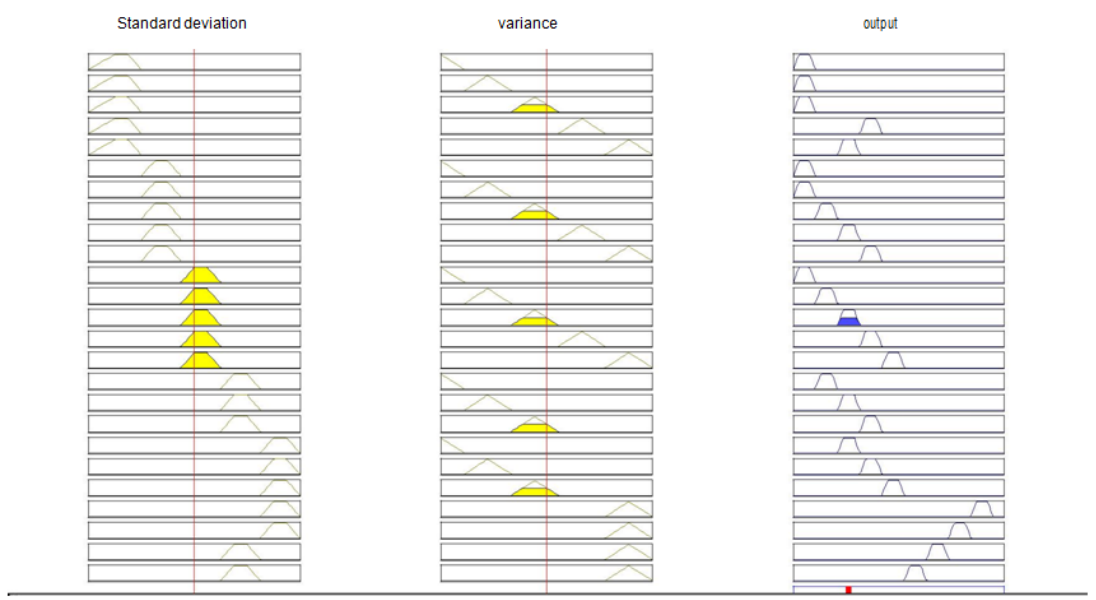

Figure 3.Rule viewer of fuzzy expert system

The brief rule sets of fuzzy expert system are given below:

1) If (Standard deviation is VSTD) and (variances is VV) then (output is INTERRUPTION).

2) If (Standard deviation is VSTD) and (variances is SV) then (output is INTERRUPTION).

3) If (Standard deviation is VSTD) and (variances is NV) then (output is INTERRUPTION). 
4) If (Standard deviation is VSTD) and (variances is LV) then (output is SWELL).

5) If (Standard deviation is VSTD) and (variances is VLV) then (output is NORMAL).

6) If (Standard deviation is SSTD) and (variances is VV) then (output is INTERRUPTION).

7) If (Standard deviation is SSTD) and (variances is SV) then (output is INTERRUPTION).

8) If (Standard deviation is SSTD) and (variances is NV) then (output is SAG).

9) If (Standard deviation is SSTD) and (variances is LV) then (output is NORMAL).

10) If (Standard deviation is SSTD) and (variances is VLV) then (output is SWELL).

11) If (Standard deviation is NSTD) and (variances is VV) then (output is INTERRUPTION).

12) If (Standard deviation is NSTD) and (variances is SV) then (output is SAG).

13) If (Standard deviation is NSTD) and (variances is NV) then (output is NORMAL).

14) If (Standard deviation is NSTD) and (variances is LV) then (output is SWELL).

15) If (Standard deviation is NSTD) and (variances is VLV) then (output is HARMONICS).

16) If (Standard deviation is LSTD) and (variances is VV) then (output is SAG).

17) If (Standard deviation is LSTD) and (variances is SV) then (output is NORMAL).

18) If (Standard deviation is LSTD) and (variances is NV) then (output is SWELL).

19) If (Standard deviation is LSTD) and (variances is VLV) then (output is SAG WITH HARMONICS).

20) If (Standard deviation is LSTD) and (variances is VLV) then (output is SWELL WITH HARMONICS).

21) If (Standard deviation is VLSTD) and (variances is VV) then (output is NORMAL).

22) If (Standard deviation is VLSTD) and (variances is SV) then (output is SWELL).

23) If (Standard deviation is VLSTD) and (variances is NV) then (output is HARMONICS).

24) If (Standard deviation is VLSTD) and (variances is VLV) then (output is FLICKER).

25) If (Standard deviation is VLSTD) and (variances is VLV) then (output is NOTCH).

\section{Classification Stage}

The proposed fuzzy expert system used to analyze and classify the eight types of power quality disturbances. The extracted input features through the S-Transform are applied as inputs to the fuzzy expert system in order to classify the disturbances. Fuzzy logic with the rule based expert system has emerged the classification tool for PQ events.

\subsection{Flowchart of the Proposed Method}

The flowchart for the Classification of Power Quality disturbances is shown in below. It has three different blocks.

- Block-(a) - Features extraction - Standard deviation and Variances are extracted

- Block-(b) - Detection and classification of the power quality disturbances

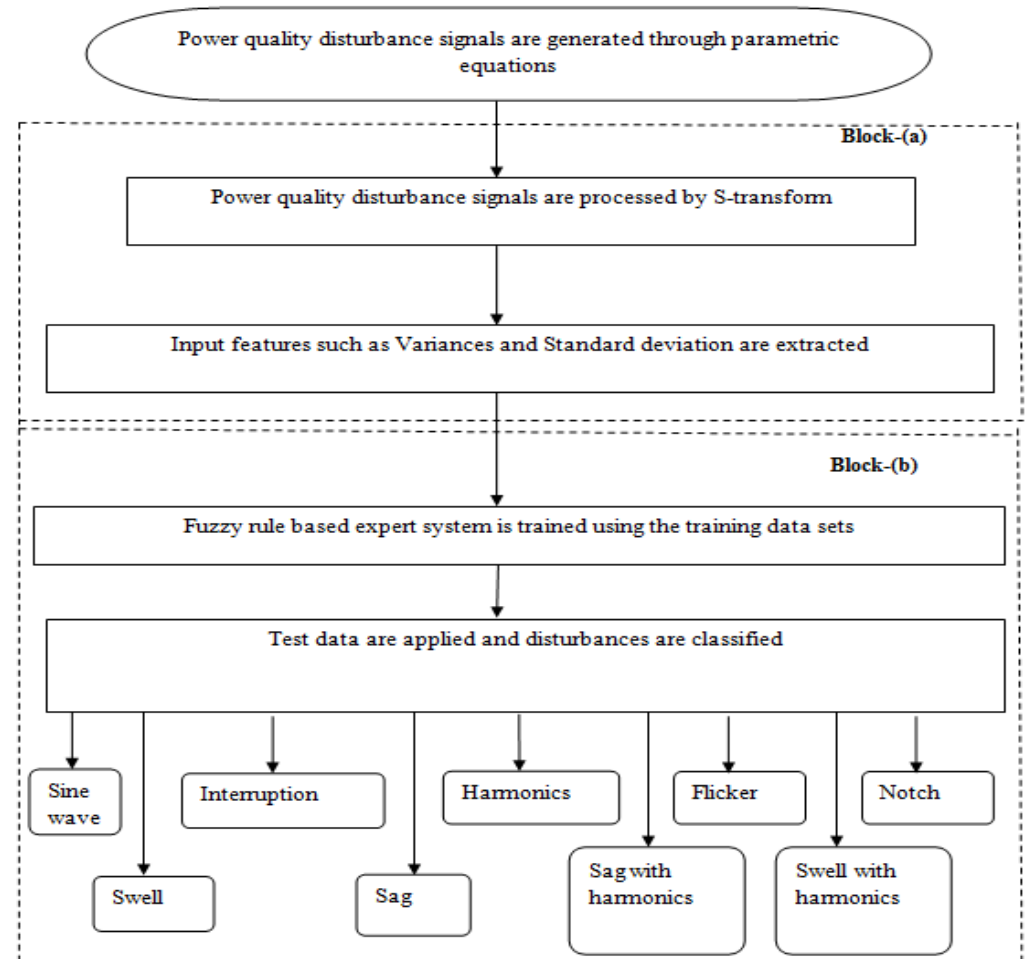

Figure 2 Flowchart for the Classification of Power Quality disturbances 


\section{Simulation And Test Results}

A set of parametric equations were used to Test data for various classes of disturbances and the signals closer to real situation can be simulated. The nine types of different power quality disturbances, namely pure sine (normal), sag, swell, outage, harmonics, sag with harmonic, swell with harmonic, notch and flicker were considered. Signal generation models and their control parameters are shown in table 1.

Table 1 Power Quality Disturbance Model

\begin{tabular}{|c|c|c|c|c|}
\hline $\begin{array}{l}\text { S1.N } \\
\text { o }\end{array}$ & $\begin{array}{l}\mathrm{PQ} \\
\text { Events }\end{array}$ & $\begin{array}{l}\text { Class } \\
\text { Symbol }\end{array}$ & Model & Parameters \\
\hline 2 & Sag & S2 & $\mathrm{f}(\mathrm{t})=\mathrm{A}\left(1-\alpha\left(\mathrm{u}\left(\mathrm{t}-t_{1}\right)-\mathrm{u}\left(\mathrm{t}-t_{2}\right)\right)\right) \sin (\omega \mathrm{t})$ & $0.1 \leq \alpha \leq 0.9 ; \mathrm{T} \leq t_{2}-t_{1} \leq 9 \mathrm{~T}$ \\
\hline 4 & Outage & S4 & $\mathrm{f}(\mathrm{t})=\mathrm{A}\left(1-\alpha\left(\mathrm{u}\left(\mathrm{t}-t_{1}\right)-\mathrm{u}\left(\mathrm{t}-t_{2}\right)\right)\right) \sin (\omega \mathrm{t})$ & $0.9 \leq \alpha \leq 1 ; \mathrm{T} \leq t_{2}-t_{1} \leq 9 \mathrm{~T}$ \\
\hline 6 & $\begin{array}{l}\text { Sag and } \\
\text { Harmonics }\end{array}$ & S6 & $\begin{array}{l}\mathrm{f}(\mathrm{t})=\mathrm{A}\left(1-\alpha\left(\mathrm{u}\left(\mathrm{t}-t_{1}\right)-\mathrm{u}\left(\mathrm{t}-t_{2}\right)\right)\right) \\
\left(\alpha_{1} \sin (\omega \mathrm{t})+\alpha_{3} \sin (3 \omega \mathrm{t})+\alpha_{5}\right. \\
\sin (5 \omega \mathrm{t}))\end{array}$ & $\begin{array}{l}0.1 \leq \alpha \leq 0.9 ; \mathrm{T} \leq t_{2}-t_{1} \leq 9 \mathrm{~T} \\
0.05 \leq \alpha_{3} \leq 0.15 ; 0.05 \leq \alpha_{5} \leq 0.15 ; \sum \alpha_{i}^{2}=1\end{array}$ \\
\hline 7 & $\begin{array}{l}\text { Swell and } \\
\text { Harmonics }\end{array}$ & S7 & $\begin{array}{l}\mathrm{f}(\mathrm{t})=\mathrm{A}\left(1+\alpha\left(\mathrm{u}\left(\mathrm{t}-t_{1}\right)-\mathrm{u}\left(\mathrm{t}-t_{2}\right)\right)\right) \\
\left(\alpha_{1} \sin (\omega \mathrm{t})+\alpha_{3} \sin (3 \omega \mathrm{t})+\alpha_{5} \sin (5 \omega \mathrm{t})\right)\end{array}$ & $\begin{array}{l}0.1 \leq \alpha \leq 0.8 ; \mathrm{T} \leq t_{2}-t_{1} \leq 9 \mathrm{~T} \\
0.05 \leq \alpha_{3} \leq 0.15 ; 0.05 \leq \alpha_{5} \leq 0.15 \\
\sum \alpha_{i}^{2}=1\end{array}$ \\
\hline
\end{tabular}

Features extracted from these input signals are applied to the fuzzy expert system to get an accurate classification of disturbances. The PQ disturbance signals generated using the Matlab based parametric equations. The following case studies are presented to highlight the suitability of the application of the proposed method.

Pure sine wave is the normal voltage signal of at frequency $50 \mathrm{~Hz}$ and its waveform is as shown in the figure 3(a).The time frequency analysis of S-transform and spectrogram representation of the pure sine wave are shown in the figures 3(b) and 3(c). The standard deviation and variances of the pure sine wave are shown in the figures $3(\mathrm{~d})$ and $3(\mathrm{e})$.

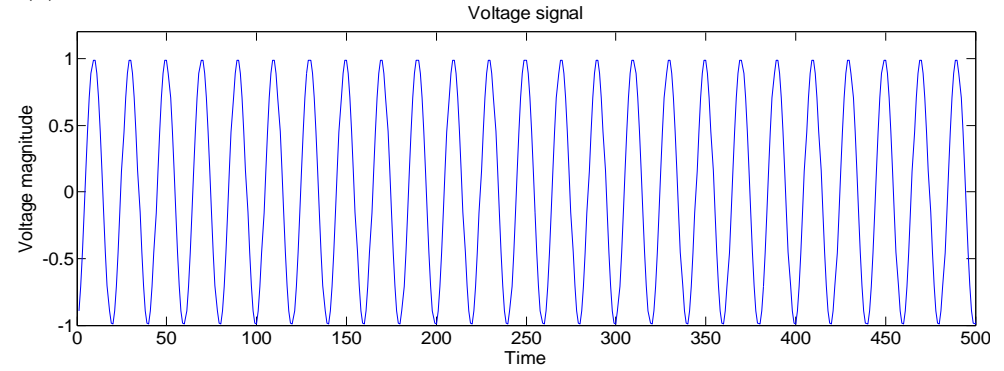

Figure 3(a)

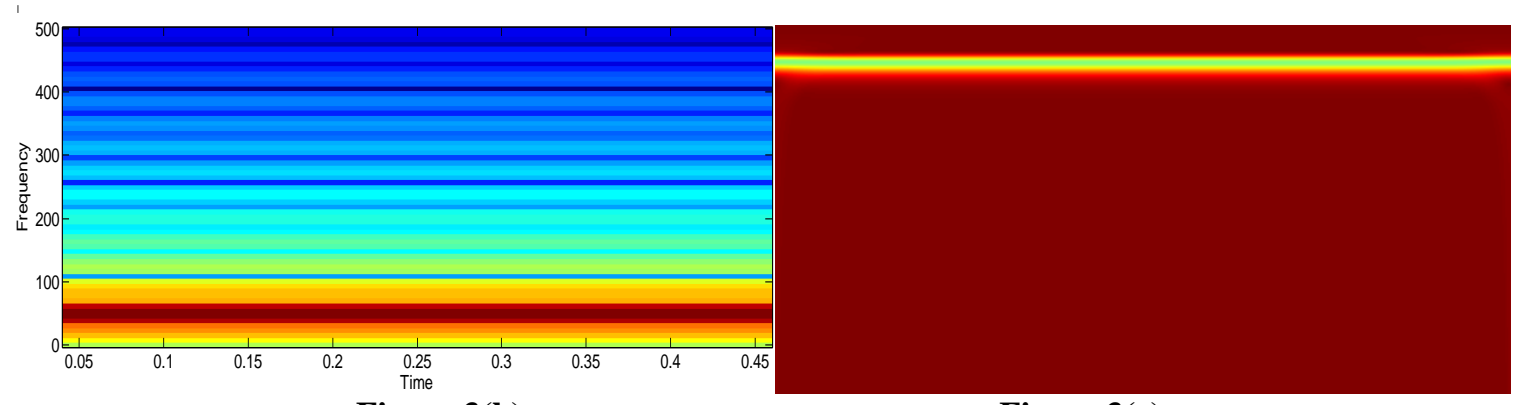

Figure 3(b)

Figure 3(c) 


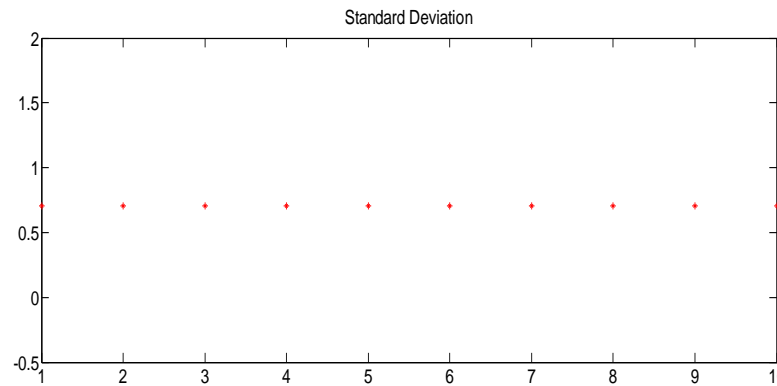

Figure 3(d)

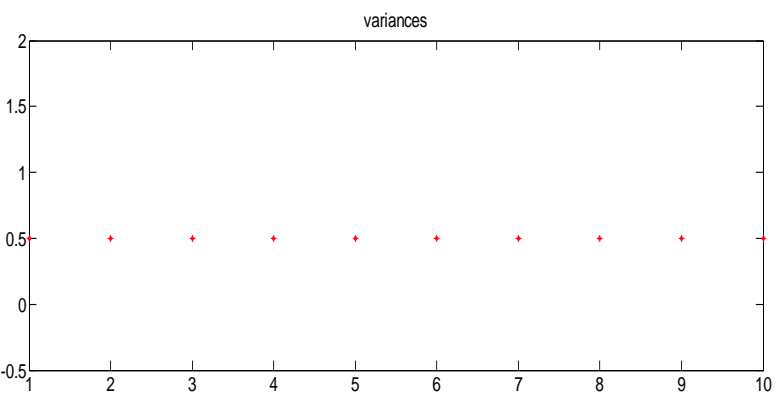

Figure 3(e)

Voltage sag (or) voltage dips causes the decrease of system voltage and its waveform is as shown in the figure 4(a).The time frequency analysis of S-transform and spectrogram representation of the voltage sag are shown in the figures 4(b) and 4(c). The standard deviation and the variances of the voltage sag are shown in the figures 4(d) and 4(e).

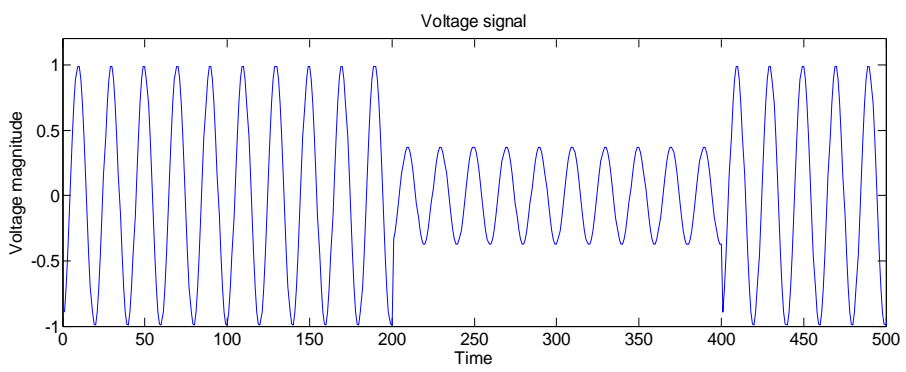

Figure 4(a)

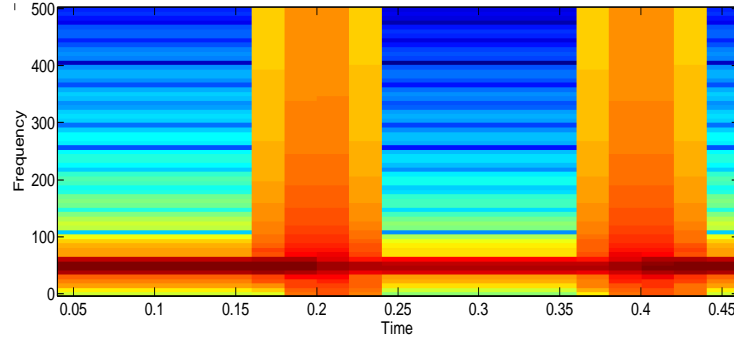

Figure 4(b)

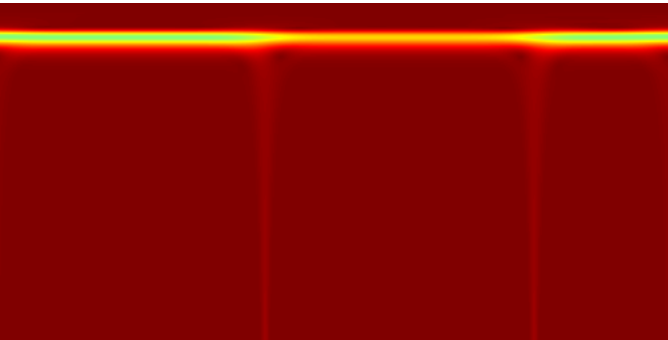

Figure 4(c)
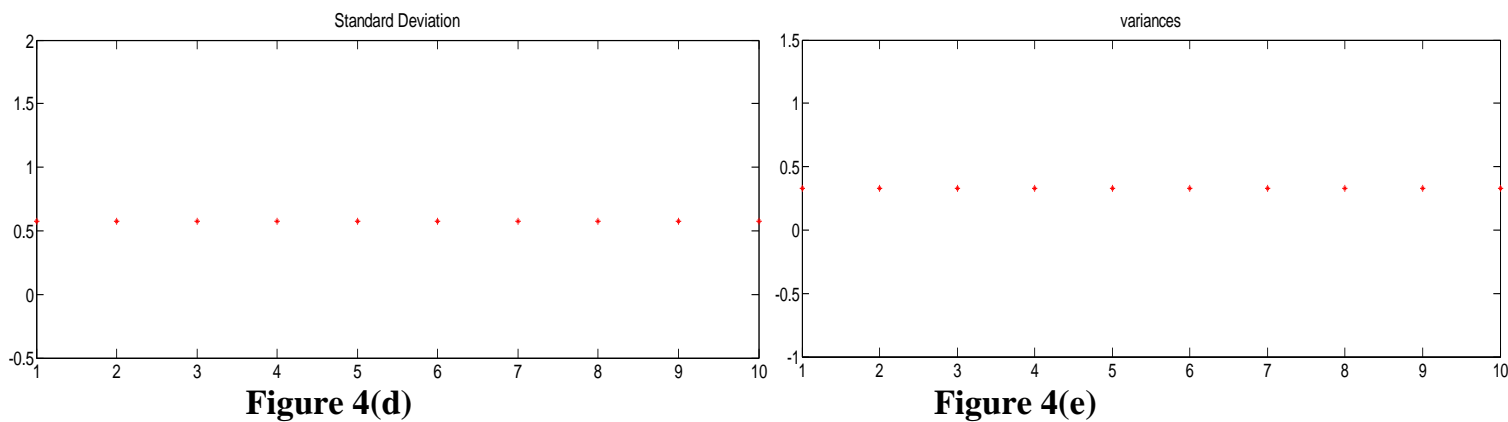

Voltage swell causes the rise of system voltage and its waveform is as shown in the figure 5(a).The time frequency analysis of S-transform representation and spectrogram of the voltage swell are shown in the figures 5(b) and 5(c). The standard deviation and the variances of the voltage swell are shown in the figures 5(d) and $5(\mathrm{e})$. 


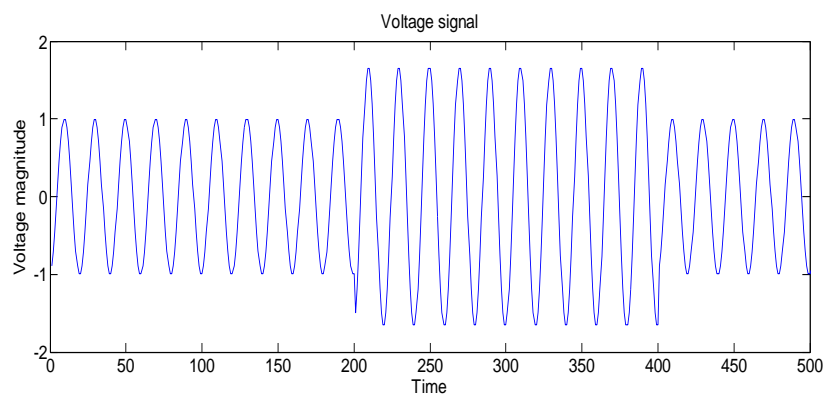

Figure 5(a)

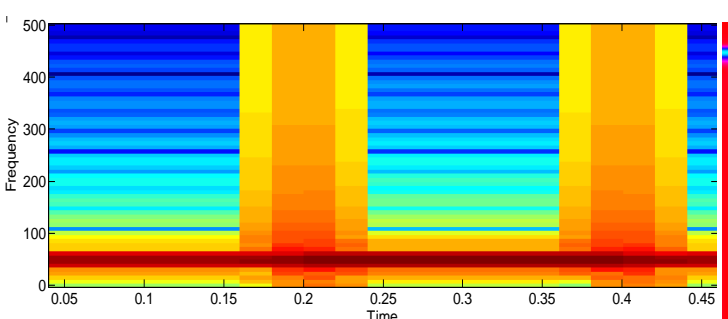

Figure 5(b)

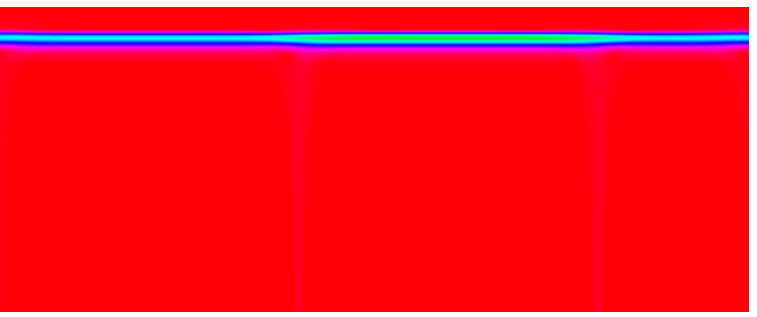

Figure 5(c)

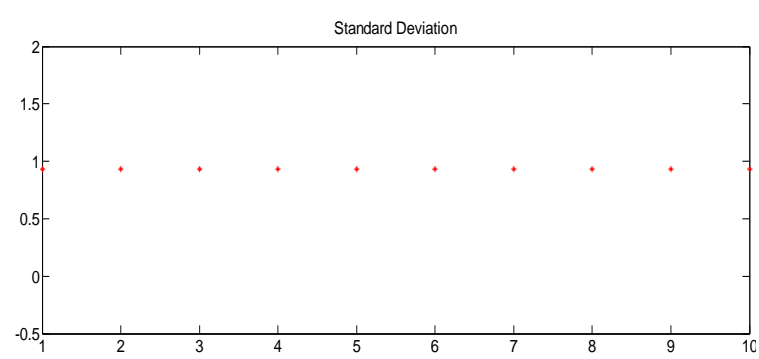

Figure 5(d)

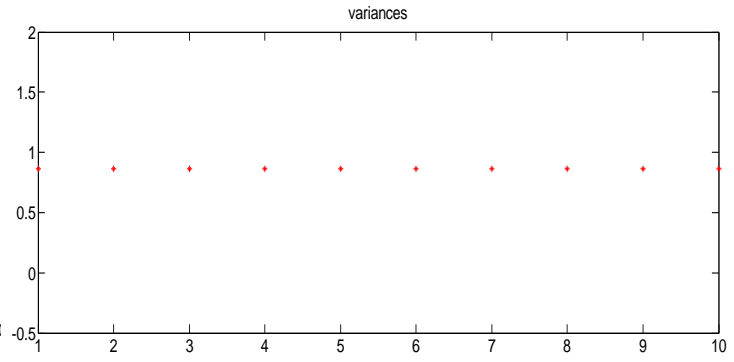

Figure 5(e)

Outages may be seen as a loss of voltage on the system for the short duration and its waveform is as shown in the figure 6(a). The time frequency analyses of S-transform representation spectrogram and of the voltage swell are shown in the figures $6(\mathrm{~b})$ and $6(\mathrm{c})$. The standard deviation, peak value and the variances of the voltage swell are shown in the figures $6(\mathrm{~d})$ and $6(\mathrm{e})$.

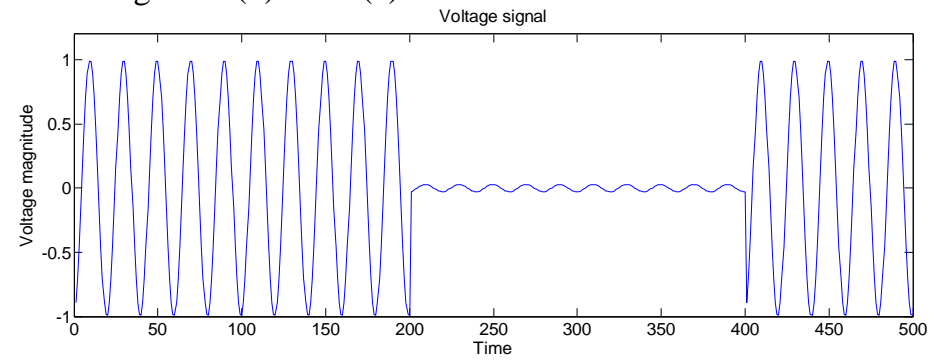

Figure 6(a)

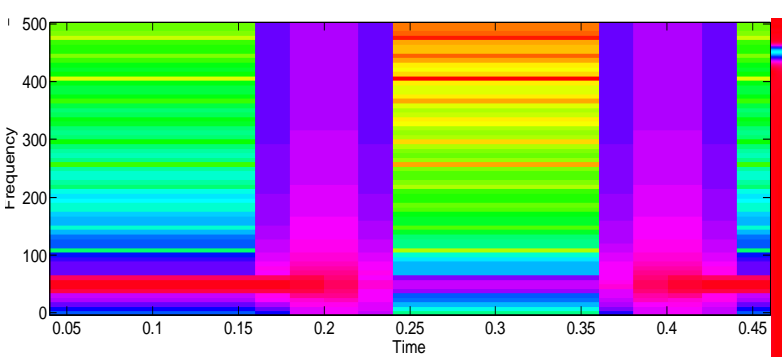

Figure 6(b)

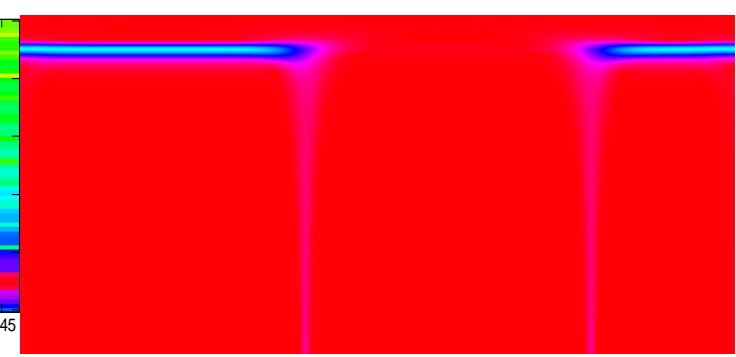

Figure 6(c) 


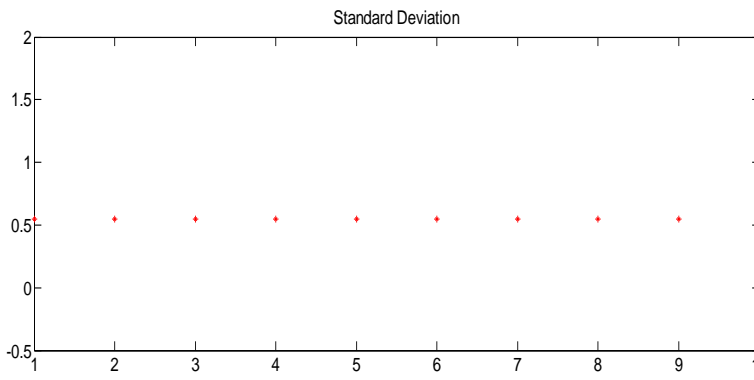

Figure 6(d)

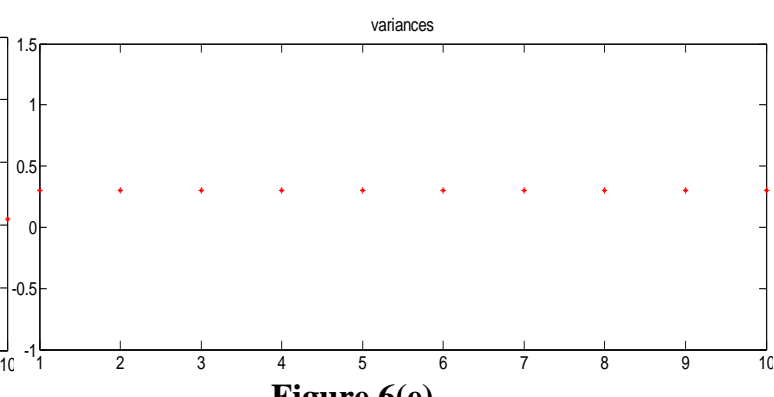

Figure 6(e)

Harmonics are generated by the connection of non linear load to the system duration and its waveform is as shown in the figure 7(a). The time frequency analysis of S-transform representation and spectrogram of the harmonics are shown in the figures 7(b) and 7(c). The standard deviation, peak value and variances of harmonics are shown in the figures $7(\mathrm{~d})$ and $7(\mathrm{f})$.

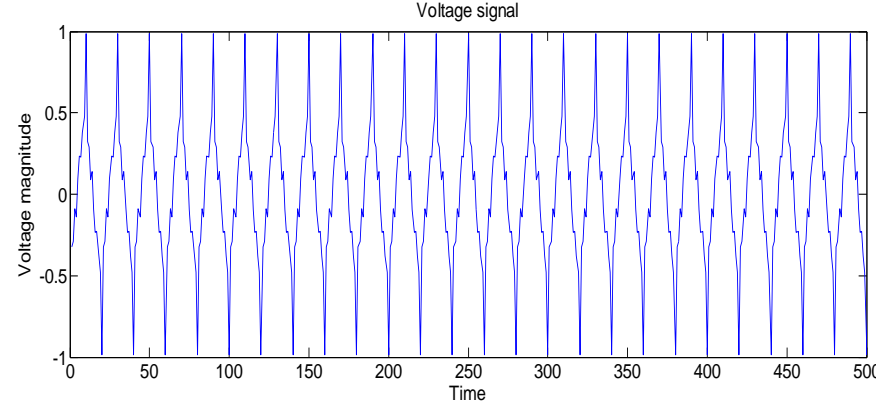

Figure 7(a)

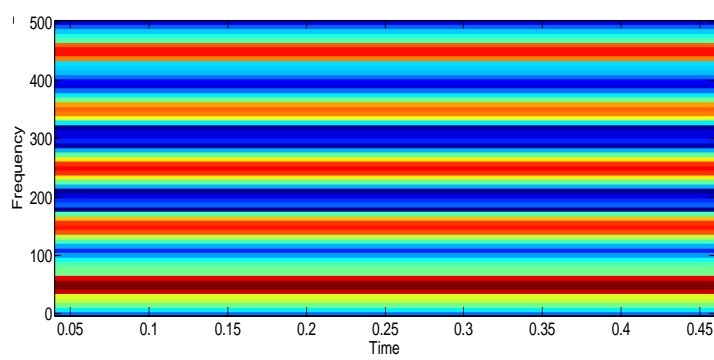

Figure 7(b)

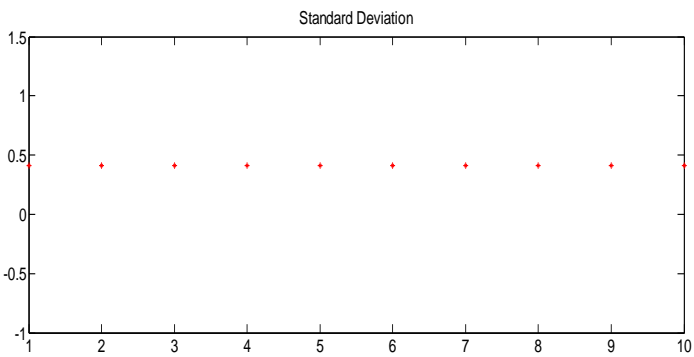

Figure 7(d)

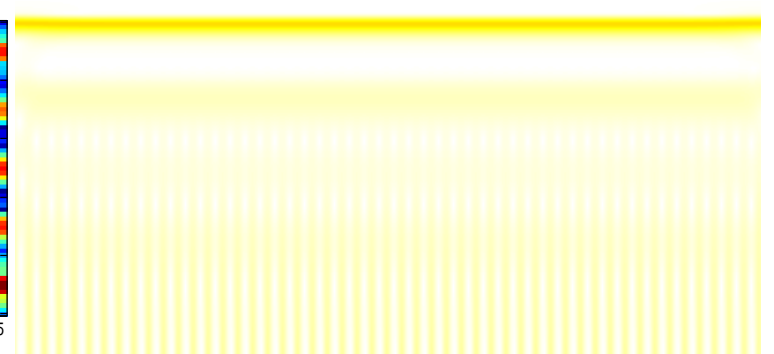

Figure 7(c)

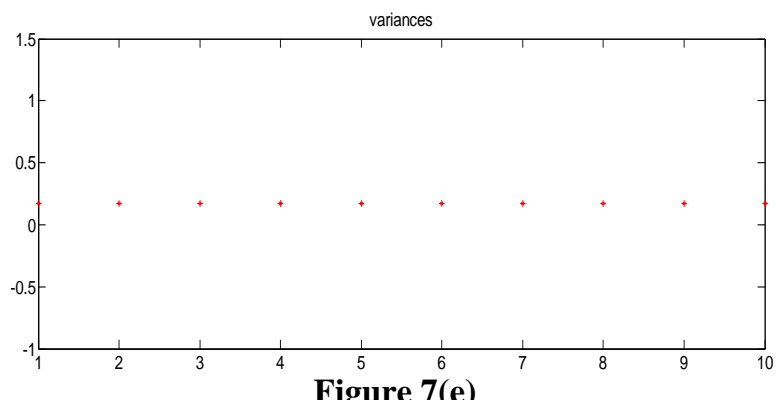

Figure 7(e)

Sag with harmonics is caused by the presence of a nonlinear load and its waveform is as shown in the figure $8(\mathrm{a})$. The time frequency analysis of S-transform representation and spectrogram of the harmonics are shown in the figures $8(\mathrm{~b})$ and $8(\mathrm{c})$. The standard deviation and the variances of the harmonics are shown in the figures $8(\mathrm{~d})$ and $8(\mathrm{e})$. 


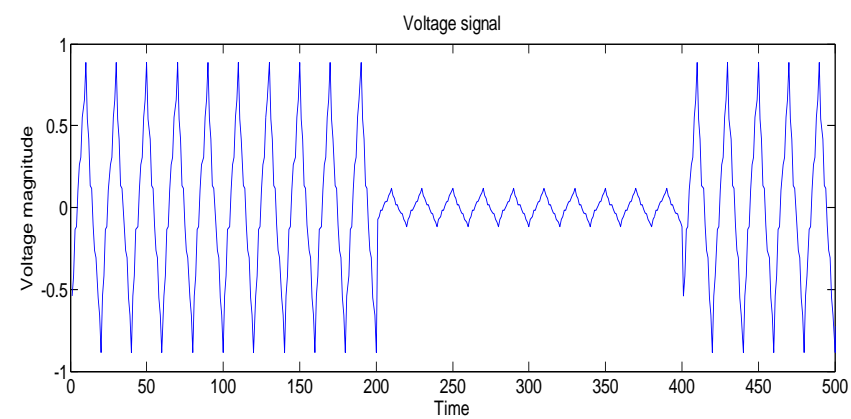

Figure 8(a)

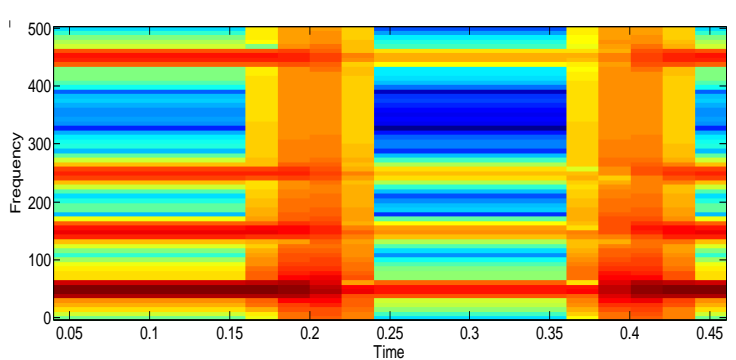

Figure 8(b)

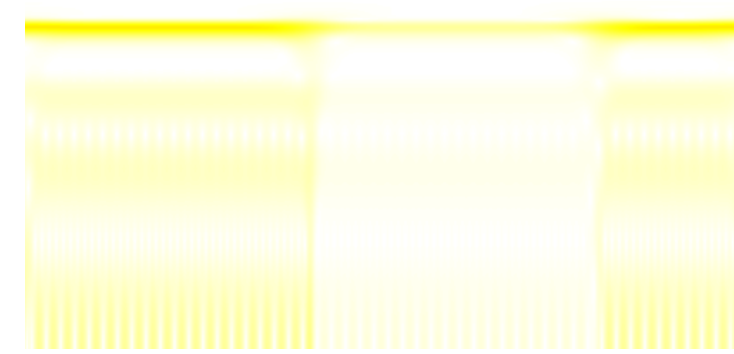

Figure 8(c)

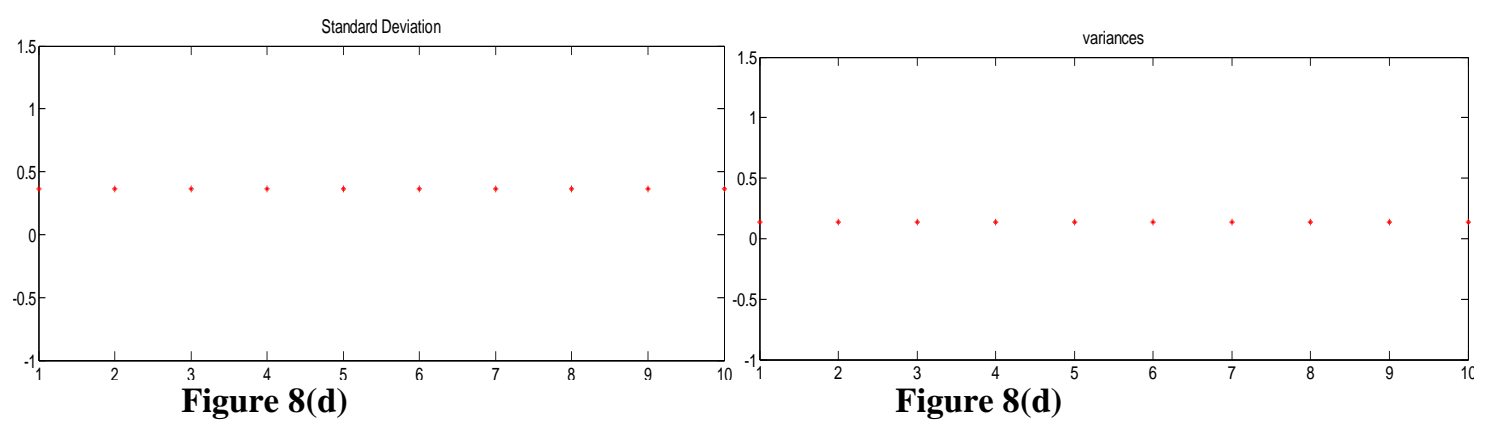

Swell with harmonics is caused by the presence of nonlinear load and its waveform is as shown in the figure 9(a). The time frequency analysis of S-transform representation spectrogram and of the swell with harmonics is shown in the figures 9(b) and 9(c). The standard deviation and variances of the swell with harmonics are shown in the fig $9(\mathrm{~d}) \& 9(\mathrm{e})$.

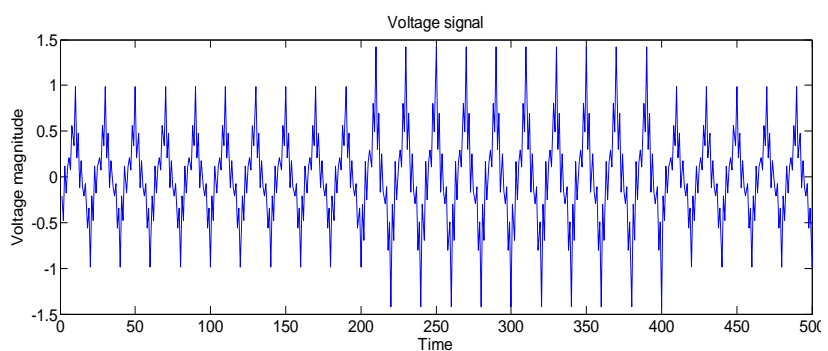

Figure 9(a)

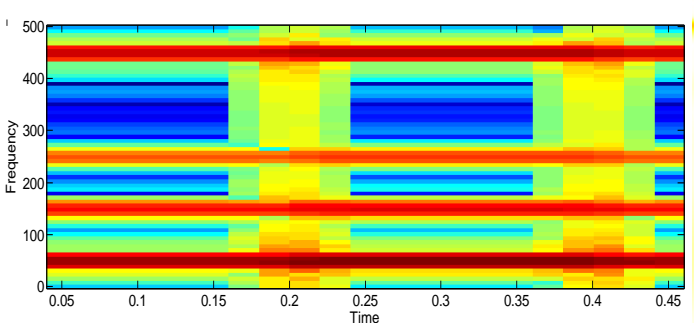

Figure 9(b)
Figure 9(c) 


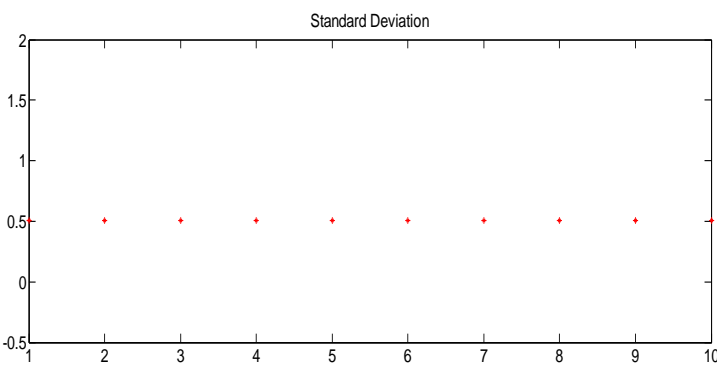

Figure 9(d)

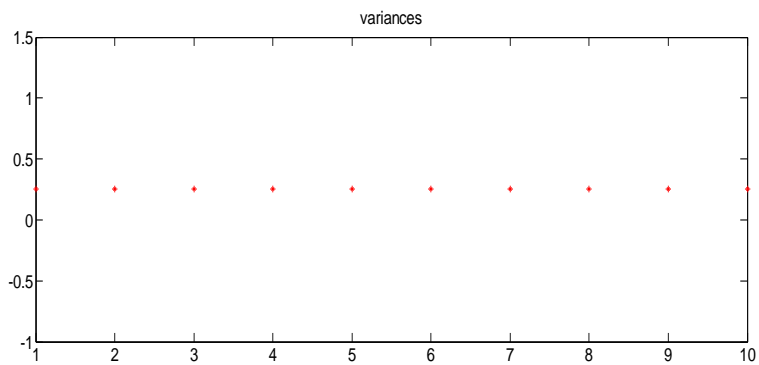

Figure 9(e)

Flicker is caused by the continuous and rapid variation of the system load and its waveform is as shown in the figure 10(a). The time frequency analysis of S-transform representation spectrogram and of the flicker is shown in the figures 10(b) and 10(c). The standard deviation, and the variances of the flicker are shown in the figures $10(\mathrm{~d})$ and $10(\mathrm{e})$.

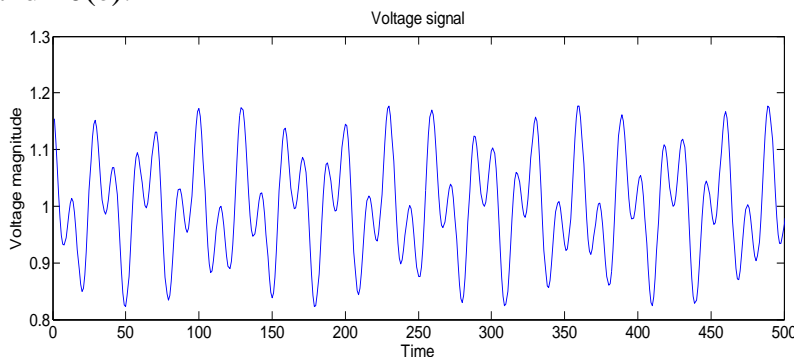

Figure 10(a)

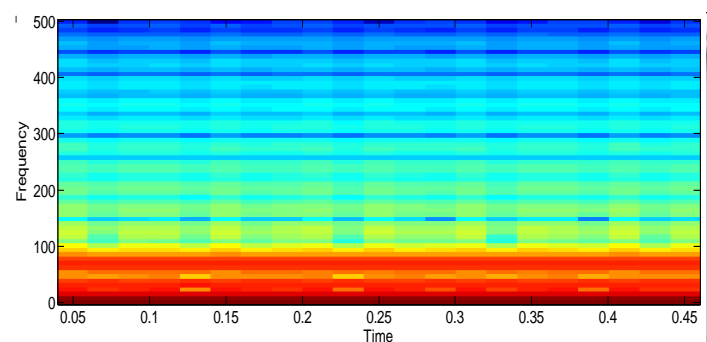

Figure 10(a)

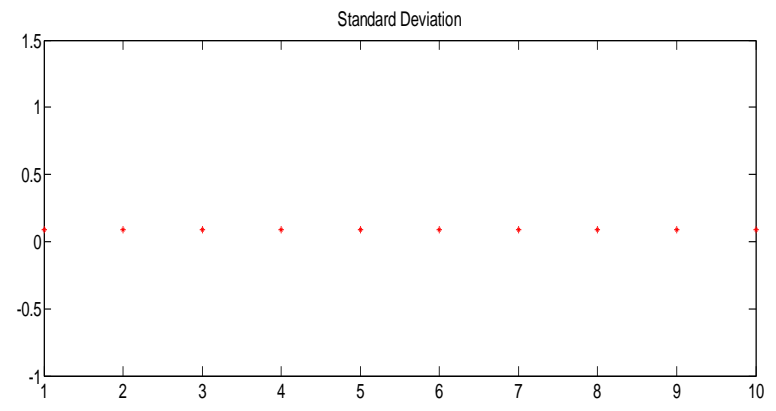

Figure 10(c)

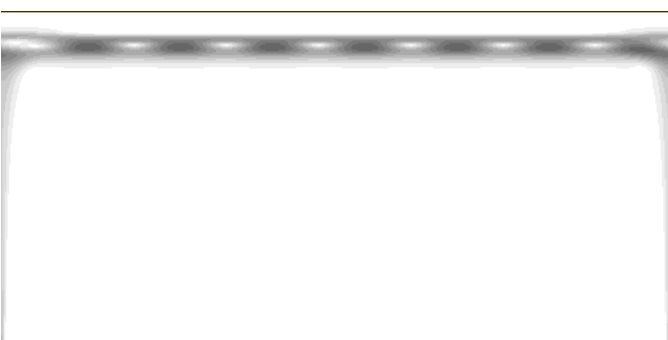

Figure 10(b)

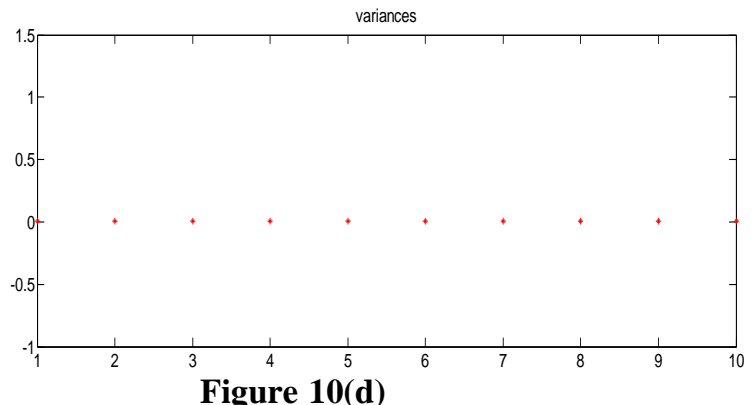

Notch is a disturbance of the nominal power voltage waveform lasting for less than half a cycle. The disturbance is initially of opposite polarity and hence it is to be subtracted from the waveform and shown in the figure 11(a). The time frequency analysis of S-transform representation spectrogram and of the notch is shown in the figures 11(b) and 11(c). The standard deviation, and variances of notch are shown in the figures 11(d) and 11(e). 


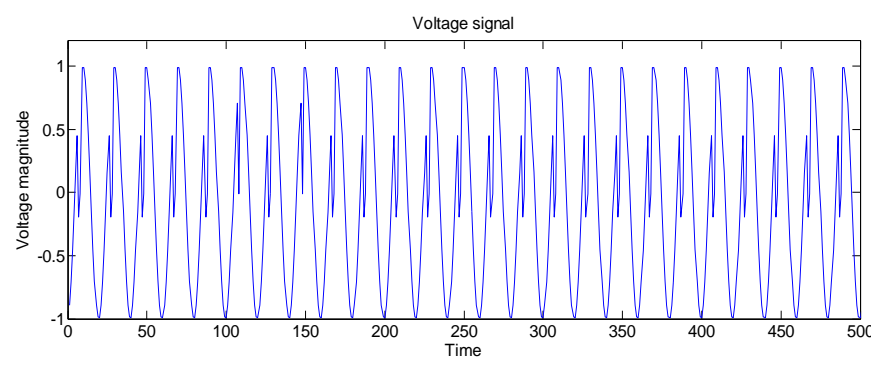

Figure 11(a)

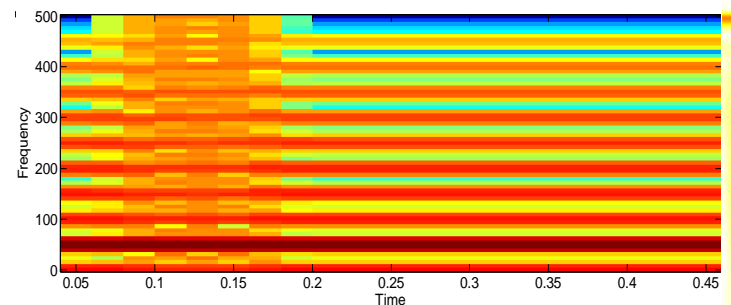

Figure 11(a)

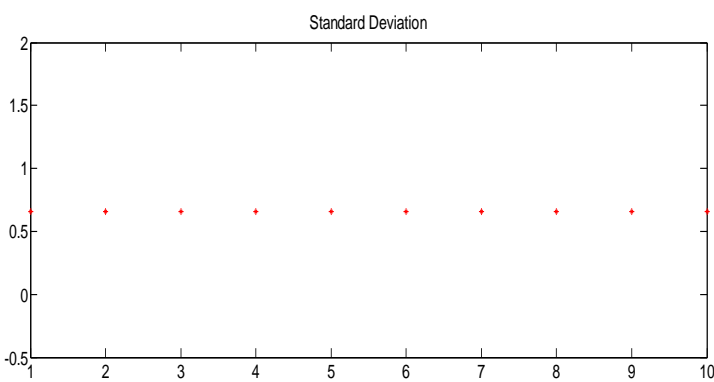

Figure 11(c)

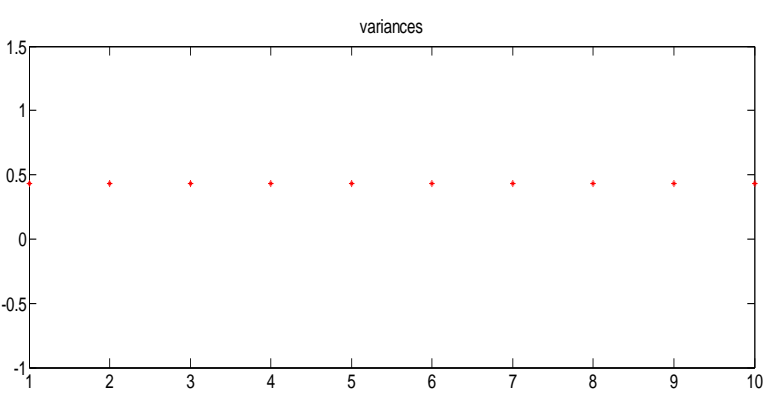

Figure 11(d)

Table 3.Classification accuracy

\begin{tabular}{|c|c|c|c|c|}
\hline \multirow[t]{2}{*}{ Sno } & \multirow[t]{2}{*}{ Power Quality Disturbances } & \multicolumn{3}{|c|}{ Percentage of Accuracy } \\
\hline & & Input Features & $\begin{array}{l}\text { S-transform based fuzzy } \\
\text { expert system }\end{array}$ & $\begin{array}{l}\text { S-transform based } \\
\text { Neural network }\end{array}$ \\
\hline 1 & Pure Sine & 100 & 100 & 100 \\
\hline 2 & Voltage Sag & 100 & 98 & 96 \\
\hline 3 & Voltage Swell & 100 & 98 & 100 \\
\hline 4 & Outages & 100 & 98 & 98 \\
\hline 5 & Harmonics & 100 & 100 & 98 \\
\hline 6 & Sag with Harmonics & 100 & 98 & 100 \\
\hline 7 & Swell with Harmonics & 100 & 100 & 97 \\
\hline 8 & Flicker & 100 & 100 & 100 \\
\hline 9 & Notch & 100 & 98 & 100 \\
\hline \multicolumn{3}{|c|}{ Overall accuracy } & 98.89 & 98.77 \\
\hline
\end{tabular}

\section{Conclusion}

In this paper, a new technique to characterize, localize and classify the various types of Power quality disturbances using S-transform and Fuzzy expert system has been proposed. The Power quality disturbance waveforms were generated through parametric equations. By using S-Transform the input features such as standard deviation and variances were extracted and Fuzzy expert system has been applied for classifying the various power quality disturbances. The classification accuracy has been validated by comparing the results obtained by the proposed technique against S-Transform based neural classifiers. The proposed technique enables the accurate classification of all nine types of PQ disturbances. Simulation results demonstrate that the performance and accuracy of the S-transform.. The results show that the proposed technique performs very well in characterization and classification of power quality disturbances.

\section{References}

[1]. Surya Santoso, Edward J. Powers, and W. Mack Grady, "Electric power quality disturbances detection using wavelet transform analysis", IEEE Transaction, 1994. 
[2]. David C. Robertson Octavia I. Camps, Jeffrey S. Mayer, William B. Gish, "Wavelet and electromagnetic power system transients", IEEE Transaction on power delivery, Vol 11, April 1996.

[3]. G.T.Heydt, A.W.Galli, "Transient power quality problem analyzed using wavelets", IEEE Transaction on power delivery, Vol 12, April 1997.

[4]. Paul S. Wright, "Short time Fourier transforms and wigner-ville distributions applied to the calibration of power frequency harmonic analyzers", IEEE Transaction on instrumentation and measurement, Vol 48, no.2, April 1999.

[5]. G.T.Heydt, P.S.Fjeld, C.C.Liu, D.Pierce, L.Tu,G.Hensley, "Applications of the windowed FFT to Electric Power quality assessment", IEEE Transaction on power delivery, oct 1999.

[6]. Surya Santoso, Edward J. Powers, W. Mack Grady, and Antony C. Parsons, "Power quality disturbance waveform recognition using wavelet based neural classifier-part 1: theortical foundation”, IEEE Transaction on power delivery, Vol 15, no.1, January 2000.

[7]. A.M. Gaouda, S.H. Kanoun and M.M.A. Salama, "On-line disturbance classification using nearest neighbor rule", Electric power systems research, 2001.

[8]. P.K. Dash, B.K. Panigrahi, G. Panda, "Power quality analysis using S-transform",IEEE Trans action of Power Delivery,2003.

[9]. M. V. Chilukuri and P. K. Dash, "Multi resolution S-transform-based fuzzy recognition system for power quality events", IEEE Transaction on power delivery, Vol 19, no.1, January 2004.

[10]. Dogan gokhan ece, and Omer nezih gerek, "Power quality event detection using joint 2-D-wavelet subspaces", IEEE Transaction on instrumentation and measurement, Vol 53, no.4, August 2004

[11]. Haibo He, Janusz A.Starzyk, A self organizing learning array system for power quality classification based on wavelet transform , IEEE Transaction on power deliv, Vol 21, no.1, January 2006.

[12]. Fengzhan Zhao, Rengang Yang, "Power quality disturbance recognition using S-transform", IEEE transaction on power delivery, Vol 22, no.2, April 2007.

[13]. C.N.Bhende, S.Mishra, B.K.Panigrahi, "Detection and classification of power quality disturbances using S-transform and modular neural network, Electric power systems research, 2008.

[14]. P.K. Dash, B.K. Panigrahi, and Birendra Biswal, "Power quality disturbance classification using Fuzzy C-means algorithm and Adaptive particle swarm optimization”, IEEE Transaction on power delivery, Vol 56, no.1, January 2009.

[15]. Thai Nguyen, yuan Liao, "Power quality disturbance classification utilizing S-transform and binary feature matrix method", Electric power systems research, 2009.

[16]. Stuti Shukla, S.Mishra, and Bhim singh, "Empirical mode decomposition with hilbert transform for power quality assessment", IEEE Transactions on Power Delivery, Vol 24, no.4, October 2009.

[17]. T.Jayasree, D.Devaraj, R.Sukanesh," Power quality disturbance classification using Hilbert transform and RBF networks", Electric power systems, 2010.

[18]. N.Rathina Prabha, N.S. Marimuthu, C.K. Babulal, “Adaptive neuro-fuzzy inference system based representative quality power factor for power quality assessment", Electric power systems, 2010.

[19]. A. Abdelsslam, Azza Eldesouky, Abdelhay A. Sallam, "Characterization of power quality disturbances using hybrid technique of linear kalman filter and fuzzy - expert system", Electric power system research,2012.

[20]. Mohammad Jasa Afroni, Danny Sutanto and David Stirling, "Analysis of Nonstationary Power-Quality waveforms using iterative Hilbert Huang transform and SAX algorithm",IEEE Transaction on power delivery, Oct 2013. 